\title{
Anatomo-Physiologic Basis for Auricular Stimulation
}

\author{
Beniamina Mercante, $\mathrm{PhD},{ }^{*}$ Francesca Ginatempo, MSc, ${ }^{*}$ Andrea Manca, $\mathrm{PhD},{ }^{*}$ \\ Francesco Melis, MD, PhD, Paolo Enrico, PhD, and Franca Deriu, MD, PhD
}

\begin{abstract}
Introduction: Stimulation of cranial nerves modulates central nervous system (CNS) activity via the extensive connections of their brainstem nuclei to higher-order structures. Clinical experience with vagus-nerve stimulation (VNS) demonstrates that it produces robust therapeutic effects, however, posing concerns related to its invasiveness and side-effects.

Discussion: Trigeminal nerve stimulation (TNS) has been recently proposed as a valid alternative to VNS. The ear presents afferent vagus and trigeminal-nerve distribution; its innervation is the theoretical basis of different reflex therapies, including auriculotherapy. An increasing number of studies have shown that several therapeutic effects induced by invasive VNS and TNS, can be reproduced by noninvasive auricular-nerve stimulation. However, the sites and neurobiologic mechanisms by which VNS and TNS produce their therapeutic effects are not clear yet.
\end{abstract}

Conclusions: Accumulating evidence suggests that VNS and TNS share multiple levels and mechanisms of action in the CNS.

Keywords: vagus nerve stimulation, trigeminal nerve stimulation, auricular stimulation, neuromodulation, brainstem, forebrain, central nervous system

\section{INTRODUCTION}

A NUMBER OF INVASIVE AND NONINVASIVE brainstimulation techniques are currently used in clinical neurology and psychiatry to modulate the activity of the central nervous system (CNS). ${ }^{1-9}$ Importantly, whatever the method used, neurostimulation has an impact on neurotransmitter release, with consequent excitation or inhibition of neuron firing, which, in turn, affects neurotransmitters' concentration in specific regions of the brain. As a result, neurostimulation modifies the intrinsic properties of neuronal membranes and synapses, development of new circuits, and the response of an individual synapse to a given synaptic drive, thus interfering with neuroplasticity. ${ }^{10}$ Neuromodulation does not necessarily convey excitation or inhibition from one neuron to another neuron, but, rather, alters the cellular and/or synaptic properties of neurons. ${ }^{11}$
By changing these properties, neuromodulatory substances reshape the output of an individual circuit, sometimes effectively rewiring or reprogramming it and thereby causing that circuit to be multifunctional.

The therapeutic application of neurostimulation includes the use of central and peripheral methods, which operate via different mechanisms. The former work by stimulating the brain directly (e.g., deep brain stimulation, transcranial direct current stimulation, repetitive transcranial magnetic stimulation), while the latter act indirectly through stimulation of peripheral nerves, such as the vagus and trigeminal nerves. In particular, the first group of techniques are supposed to operate with a top-down mechanism that primarily modulates the activity of cortical networks and secondarily induces subcortical excitability changes. Yet, stimulation of peripheral nerves may affect brain activity through a bottom-up mechanism, for instance, by stimulating cranial

Department of Biomedical Sciences, University of Sassari, Viale San Pietro 43/b, 07100 Sassari Italy.

*These authors contributed equally to this article. 
nerve nuclei in the brainstem, which, in turn, make extensive connections to higher CNS structures. ${ }^{12,13}$

With specific regard to peripheral methods, studies on vagus- and trigeminal-nerve stimulations (VNS and TNS, respectively), along with clinical experience, have demonstrated that these neurostimulation modalities can produce robust therapeutic effects without incurring unsafe consequences on normal brain function.

\section{CLINICAL APPLICATIONS OF VAGUS- AND TRIGEMINAL-NERVE STIMULATIONS}

The first observation that VNS directly affects brain function was performed in cats by Bailey and Bremer, in 1938. ${ }^{14}$ This seminal work was confirmed by Dell and Olson in $1951,{ }^{15}$ and primate studies provided evidence of VNS effects on the basal limbic structures, thalamus, and cingulate cortex. ${ }^{16}$ Based on these findings, it was hypothesized that VNS might have anticonvulsant properties, ${ }^{17,18}$ with a potentially positive effect on both direct termination of an ongoing seizure as well as seizure prevention. ${ }^{19}$ Since these seminal studies, VNS has been demonstrated widely to control seizure activity across multiple species, suggesting that VNS anticonvulsant effects involve widespread neuromodulatory mechanisms that are phylogenetically wellconserved. ${ }^{20}$ Following these basic and clinical works, VNS was developed further as an adjunct treatment for seizure disorders, obtaining the approval of the U.S. Food and Drug Administration (FDA) for the treatment of pharmacoresistant epilepsy in $1997 .^{21-24}$

VNS-induced mood elevation was serendipitously observed in patients with epilepsy and prompted researchers also to examine possible effects of VNS on emotional health. ${ }^{25-27}$ Several clinical trials were conducted to evaluate the efficacy of VNS in patients whose conditions were resistant to standard antidepressant treatments. This prospective investigation of VNS effects in patients with depression resulted in the FDA approval of VNS as an adjunct therapy for the treatment of drug-resistant major depression in $2005 .^{28}$

However, clinical use of VNS has also been shown to have several limitations. ${ }^{29,30}$ Some of them are related to the invasiveness of the method, due to the surgical implantation of the stimulating device, for which hoarseness, cough, vocal-cord paralysis, and infections are regarded as common adverse events. However, the most important limitation in the clinical use of VNS is the presence of a visceral efferent component in the vagus nerve that affects cardiac function. ${ }^{31}$ Therefore, in order to avoid a possible depressive effect on cardiac performance, VNS cannot be applied bilaterally nor at high-stimulation frequencies, which limits its potential efficacy.

To overcome the limitations of VNS, during the last decade, an increasing number of experimental and clinical researchers have proposed TNS as an alternative method. In fact, this kind of neuromodulation produces similar effects to VNS but without the autonomic side-effects. Due to this feature, TNS can be delivered bilaterally with potentially greater beneficial effects obtained at lower stimulation intensities. ${ }^{32,33}$ In this light, an increasing number of studies have shown that TNS is beneficial for seizure control, depression, and migraine, and, very recently, the FDA approved a clinical trial for TNS treatment of Lennox-Gastaut syndrome. $^{34}$

In addition to epilepsy and depression, researchers have proposed a clinical application for VNS and TNS for treating variety of disorders, such as migraine, ${ }^{35}$ anxiety,${ }^{36}$ cognitive impairment, ${ }^{37,38}$ Alzheimer's disease, ${ }^{39}$ autism,${ }^{40}$ tinnitus, ${ }^{41,42}$ poststroke symptoms, ${ }^{43-45}$ post-traumatic stress disorder, ${ }^{46}$ eating disorders ${ }^{47}$ and obesity. ${ }^{48}$ Although clinical trials on the topic are accumulating, most of them are preliminary proof-ofconcept pilot studies obtained with limited sample sizes.

\section{The Vagus Nerve}

The vagus nerve is the tenth, and longest, cranial nerve, with a somatic afferent distribution to the external ear. This nerve is a major component of the parasympathetic section of the autonomic nervous system. The vagus nerve contains A-fibers, B-fibers, and C-fibers, which have different anatomical features and functional properties. ${ }^{49}$ The efferent component of the vagus nerve represents $\sim 20 \%$ of the total number of fibers contained in the nerve. This component originates from the nucleus ambiguous (NA) and the dorsal motor nucleus (DMN) of the vagus nerve and travels through the head, neck, thorax, and abdomen to exert a parasympathetic influence on multiple organs located in these body regions. From these organs, vagal afferent fibers carry general and special sensory information to the nucleus of the nucleus of the solitarii tract (NST) ${ }^{50,51}$ Moreover, sensory somatic fibers innervating a portion of the external ear project to the spinal trigeminal nucleus ( $\mathrm{spV}$ ). Thus, the NST and the $\mathrm{spV}$ are, respectively, the recipients of visceral and somatic sensations carried by the vagus nerve to the brain. These nuclei, in turn, project mono- or polysynaptically to a wide network of areas in the CNS.

\section{The Trigeminal Nerve}

In addition to providing motor innervation to masticatory muscles, the trigeminal nerve is responsible for sending tactile and nociceptive information from the face and from the anterior region of the external ear to the CNS, using A beta, A delta, and $\mathrm{C}$ afferent fibers. ${ }^{52,53}$ Afferent projections from the trigeminal ganglion ascend to the trigeminal nuclei in the brainstem. It is remarkable that that, unlike the vagus nerve, the trigeminal nerve contains no autonomic outflow fibers. However, the somatic afferents carried by the trigeminal nerve reach extratrigeminal regions in the brainstem and in the forebrain, which are important for the regulation of many autonomic functions. In fact, it has been 
shown recently that trigeminal sensory nuclei-in addition to sending tactile and pain information to the thalamus and from there to the somatosensory cortex-also project bilaterally to multiple nearby brainstem nuclei, including the NST, the locus coeruleus (LC) and the dorsal raphe nucleus (DRN). ${ }^{54}$

In addition to these brainstem regions, projections from the trigeminal sensory complex reach forebrain structures, such as the endopiriform nucleus, the entorhinal cortex, the hippocampus and the amydgala, ${ }^{54}$ which all have important physiologic roles. These data provide anatomical support to physiologic results proving that TNS affects brainstem structures $^{55,56,57}$ which, in turn, influence forebrain areas involved in the pathophysiology of specific CNS disorders, on which TNS has been shown to exert therapeutic effects. ${ }^{35,58-60}$ In this sense, the antiseizure effects of TNS seems particularly interesting, both in animal models ${ }^{33,38,54}$ and in patients with epilepsy. ${ }^{59,61-63}$

These anatomical data confirm previous physiologic findings proposing that the vagus and trigeminal nerves share common relay stations in the brainstem including the NST, $\mathrm{LC}$, and raphe nucleus $(\mathrm{RN}){ }^{64}$ These three structures seem to play a key role in the therapeutic effects of VNS and TNS.

\section{The Nucleus of the Solitarii Tract}

The NST is an important center for the regulation of visceral, cardiovascular, and respiratory functions, through control of swallowing and cardiomotor motoneurons in the NA and DMN, respectively. The caudal part of the NST also projects to the periaqueductal grey matter and to the visceral nuclei in the spinal cord, mediating visceral sensation. Visceral inputs are relayed from the NST to the expiratory and vasomotor centers situated in the reticular formation of the brain medulla.

The most abundant pathways ascending from the interoceptive portion of the NST reach the forebrain, in particular, the bed nucleus of the stria terminalis, which projects substantially to the amygdala, along the ventral "amygdalofugal" pathway. Moreover, the NST sends information concerning the internal body to the insular cortex, after making further synaptic connections with the parabrachial complex and the ventral posteromedial nucleus of the thalamus.

The insula is an area strongly interconnected with paralimbic structures, including the amygdala and the hippocampus. One needs to recall that the NA and the NST receive most of the sensitive fibers from the $\mathrm{spV}$ and that all of these structures are reciprocally connected with the area postrema, which is a medullary structure that has a key role in nausea and vomiting.

As noted before, the NST - and in particular its caudal part-is a key integrative center for reflexive control of visceral motor function, and performs important relays of visceral sensory information to other brainstem nuclei and forebrain structures, including the parabrachialis nucleus; hypothalamus; amygdala; thalamus; and the medial, prefrontal, and insular cortices.

Although one might propose that the posterior insular cortex serves as the primary visceral sensory area and that the medial prefrontal cortex serves as the primary visceral motor area, it might be more useful to emphasize the interactions among these cortical areas and the related subcortical structures mentioned above. Taken together, they constitute a central autonomic network, which accounts for the integration of visceral sensory information with input from other sensory modalities and higher cognitive centers that process semantic and emotional experiences.

\section{The Locus Coeruleus and Raphe Nucleus}

The LC and the RN, respectively, provide the noradrenergic and serotoninergic innervation at virtually all levels in the CNS, with key roles in the regulation of autonomic functions, arousal, the wake-sleep cycle, pain, anxiety, and mood. ${ }^{20,65}$

The major inputs to the LC originate from the nucleus paragigantocellularis and the perifascicular area of the nucleus prepositus hypoglossi, which make the former an excitatory connection using noradrenaline and the latter an inhibitory synapsis using $\gamma$-aminobutyric acid (GABA). Experimental studies in animals have shown that the connection between NST and the DRN is mediated by the LC, via the activation of alpha-1 postsynaptic adrenoceptors. ${ }^{66,67}$ It has been suggested that, when the vagus nerve is stimulated, the excitatory pathway from the NST to the LC is facilitated more than the inhibitory one; thus, the indirect effect exerted by the NST on the DRN, via LC, is mainly excitatory. Several experimental studies on animal models and also functional neuroimaging in humans have associated this connection with the antiseizure and antidepressant effects of VNS. ${ }^{67,68}$

\section{Neurochemical Effects and Pathophysiologic Implications of Vagus-Nerve and Trigeminal- Nerve Stimulation}

The pathways by which VNS and TNS exert their multiple effects on CNS activity are still poorly understood.

The vagus nerve primarily projects to the NST, where the nerve releases glutamate and aspartate, GABA, acetylcholine, and other neuropeptides for signal transduction.

There is a growing body of evidence implicating noradrenaline as a key mediator of VNS-induced effects in the CNS. VNS has been shown to result in a long-lasting increase in release of noradrenaline in the basolateral amygdala, in the thalamus, hippocampus, and neocortex, which is likely mediated by the LC or by a direct projection from the A2 cell group of the NST, which also projects to the LC. ${ }^{69}$ Experimental evidence links these structures and noradrenaline to the antiseizure and antidepressant effect of VNS. $^{70,71}$ VNS has also been shown to increase levels of 
free GABA in cerebrospinal fluid and in the hippocampus, as well as decreasing glutamate and increasing GABA in the NST. ${ }^{72,73}$ These effects have also been accounted for the seizure-attenuating effects of VNS. ${ }^{20}$

Modulation of the serotonergic system is another possibility, as a major projection of the NST is the DRN, which is the main source of the serotoninergic innervation of the brain. ${ }^{74}$ The function of many limbic and paralimbic regions of the brain depends on levels of noradrenaline and serotonine. ${ }^{75,76}$ This can explain, reasonably, the modulatory effects of VNS on mood, anxiety, and other neuropsychiatric disorders. ${ }^{71}$

VNS increases neurotrophic factors in both the hippocampus and neocortex, ${ }^{77-79}$ suggesting that this action may be implicated in VNS' antidepressant effect. ${ }^{80}$

VNS and TNS also stimulate $c$-Fos transcription, cellular proliferation, and neurogenesis in the hippocampus, ${ }^{54,79}$ which, along with the neurotropic effect, have been associated with VNS effects on neuronal plasticity, memory, learning, mood, and cognitive processes. ${ }^{81-83}$

Furthermore, in studies as recent as 2014, it has been shown that VNS modulates the release of a variety of factors such as nitric oxide, melanocortins, inflammations, and glutamate excitotoxicity, that are involved in the physiopathology of ischemic stroke; these actions are thought to be responsible for the reduction of stroke volume and of the perilesional edema observed in recent studies performed in poststroke animal models and in clinical observations. ${ }^{84}$

In addition, the vagus nerve mediates an anti-inflammatory effect by suppressing cytokine production via the so-called cholinergic anti-inflammatory pathway and, through action on inflammation and the serotoninergic pathway, the vagus nerve exerts an antinociceptive effect. ${ }^{85}$

Finally, there is a growing interest in the effects of VNS on factors and areas involved in food intake and metabolism, which has led to a general agreement on the beneficial effects of VNS on glucose tolerance and body weight. ${ }^{86,87}$

With respect to the physiologic mechanisms mediating the clinical effect of TNS, research is not as advanced as on those mediating VNS. Based on the anatomical and functional data reported above and on recent experimental data, ${ }^{33,38,54-56}$ it can be assumed that the antiseizure and antidepressant effects as well as the antimigraine actions of TNS might share the same mediators and CNS targets used by VNS.

\section{Transcutaneous Vagus-Nerve and Trigeminal-Nerve Stimulation}

Both the vagus and trigeminal nerves can be accessed for stimulation not only surgically but also transcutaneously, and the latter option is, of course, of great interest because of its noninvasiveness, safety, and affordability. Transcutaneous stimulation of the trigeminal nerve is commonly delivered by applying the stimulating electrodes on the skin over the emergence of the supraorbital and/or infraorbital branches of the trigeminal nerve. ${ }^{88}$ Transcutaneous stimu- lation of the vagus nerve is possible only at the level of the external ear, which is the only place on the surface of the body with afferent vagus-nerve distribution. The external ear is also innervated by trigeminal nerve fibers, which partially overlap with the vagus territory.

In more detail, the external ear has a complex innervation, characterized by a considerable overlap between multiple nerves. ${ }^{89,90}$ It is supplied by at least four nerves: (1) the auriculotemporal nerve; (2) the auricular branch of the vagus nerve (ABVN); (3) the lesser occipital nerve; and (4) the greater auricular nerve (Fig. 1). The auriculotemporal nerve stems from the mandibular branch of the trigeminal nerve, which mainly supplies the anterosuperior and anteromedial areas of the external ear. The ABVN is the only peripheral branch of the vagus nerve, and mainly supplies the auricular concha and most of the area around the auditory meatus. The lesser occipital nerve originates from C-2 branch of the cervical plexus and supplies sensory innervation for the superior third of the external ear. The greater auricular nerve originates from C-2 to C-3 branches of the cervical plexus and supplies the lower parts of the auricle.

The innervation of the ear represents the theoretical basis of different reflex therapies, including auriculotherapy. ${ }^{89}$ Based on this assumption, the literature on the therapeutic effects of auricular stimulation on the symptoms of several disorders is increasing greatly, ${ }^{91-97}$ and these studies are showing that the beneficial effects induced by invasive VNS and TNS can be reproduced by noninvasive auricular stimulation. ${ }^{98}$

Although all of anatomo-functional data reported above were obtained following invasive VNS and TNS, accumulating evidence seems to suggest that the same brainstem and forebrain areas are influenced by stimulation of the ear.

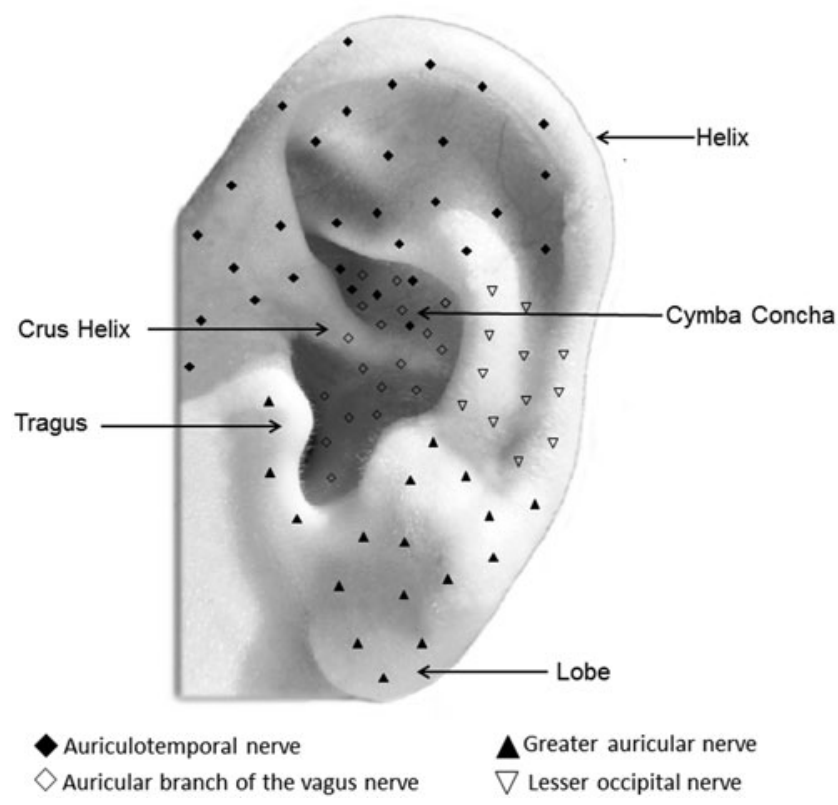

FIG. 1. Schematic representation of the innervation of the external ear. 
Neurophysiologic studies have shown that far-field potentials (i.e., potentials that are produced by neural generators located at a distance from the recording electrodes) were recorded from the scalp following auricular stimulation. These potentials, called vagus-somatosensory-evoked potentials, were detected clearly at latencies compatible with a brainstem origin, at electrode positions C-4-F-4 only after stimulation at the inner side of the tragus of the contralateral ear, but not at the other stimulation positions in the ear. ${ }^{99-101}$

Within the last decade, several fMRI studies also demonstrated that the central projections of the vagus nerve can be accessed via electrical stimulation of the external ear. ${ }^{92,94,102}$ The effects of the stimulation of the cymba concha of the external ear (i.e., the upper, smaller part of the external ear lying above the crus helicis; Fig. 1), ${ }^{90}$ were compared with those obtained by stimulation of the earlobe, taken as a control. Data have shown that the stimulation of the cymba concha induces the activation in the medulla of the spV and of the NST. In the pons, all of those regions that were seen before as main targets of VNS and TNS - such as the LC and the parabrachialis nucleus-were activated by the stimulation of the cymba concha.

In the midbrain, the periacqueductal grey matter, the DRN, the substantia nigra, and the red nucleus were the regions significantly activated by cymba concha stimulation, in comparison with the control. In the forebrain, in addition to the primary somatosensory area, the amygdala, the fornix, the thalamus, and the insula were activated consistently. Finally, data showed that the hippocampus and the hypothalamus were also targets of auricular stimulation, but the observed effect was deactivation. The findings of functional magnetic resonance imaging (fMRI) studies provided evidence that noninvasive electrical stimulation of the somatic afferent branch of the vagus nerve activated both visceral and somatic vagal projections in the brain.

In particular, stimulation of the ABVN, via the cymba concha, activates the NST, the LC, the DRN. and other primary and higher-order vagal projections in the brainstem and forebrain. It has been suggested that the patterns of activation and deactivation observed in studies performed with healthy subjects provide a point of reference for understanding the mechanism(s) underlying the anticonvulsive, antidepressive, and antinociceptive effects of transcutaneous VNS. ${ }^{94,102,103}$ Interestingly, it has been shown that the brain sites to which the vagus projects remained maximally active during the poststimulation period. Furthermore, their activity declined gradually, persisting for 11 minutes after cessation of cymba concha stimulation, as shown by a time-course analysis of the percent change of the bold signal for each significantly active region compared to baseline. ${ }^{102}$ This finding could explain the concomitant persistence of the cognitive and behavioral effects of ear stimulation. ${ }^{102}$

In rats, stimulation of the $\mathrm{ABVN}$ in the area of the auricular concha showed that transcutaneous VNS plays an important role in immunoregulation through activation of the cholinergic anti-inflammatory pathway and downregulation of proinflammatory cytokine expressions and nuclear factor kappaB (NF- $\kappa \mathrm{B}$ ) activities. ${ }^{93}$ The activation of the ABVN evoked by transcutaneous VNS, activates the NST and the integrated output is carried by the efferent vagus nerve to inhibit inflammatory responses. ${ }^{93}$ Furthermore, researchers who performed animal studies reported that auricular stimulation reduced infarct volume and induced angiogenesis after cerebral ischemia, and might, therefore, represent a new potential therapy target for stroke. ${ }^{104}$ These data, obtained in experimental animals, indicate that transcutaneus VNS treatment might improve recovery of neurologic function, ${ }^{104,105}$; however, human studies have yet to be performed.

Finally, both animal and human studies demonstrate that the vagus and trigeminal nerves project bilaterally to their target regions in the CNS, and that there is a wide central integration of visceral and somatic information carried by these nerves. Data presented so far suggest that, conversely from the invasive stimulation of the vagus nerve, there is no point in preferring one side or the other in auricular stimulation. ${ }^{106}$

\section{DISCUSSION}

The innervation of the external ear with fibers derived from the vagus and trigeminal nerves, represents the main theoretical basis for auricular stimulation. This assumption is supported by several sources of evidence including: (1) longstanding clinical experience with auricular acupuncture ${ }^{89,107}$; (2) case-control observational studies dealing with the therapeutic effects of auricular stimulation; (3) neurophysiologic and fMRI studies in humans showing that auricular stimulation modulates the functional state of brain areas that are the target of the vagus and trigeminal nerves; and (4) experimental studies with animal models, proving that auricular stimulation influence produces effects in the CNS that are comparable to those produced by invasive VNS and TNS.

Consistent evidence shows that trigeminal and vagal systems share common relay stations in the brainstem, which could explain the similar clinical effects of VNS and TNS. The NST and the spV are the recipients in the brainstem of visceral and somatic afferents carried by these nerves. These nuclei seem to play a key role in the effects of both vagal and trigeminal neuromodulation by projecting sensory information to the LC and DRN. These structures affect the function of different areas of the brain profoundly, including the amygdala, hippocampus, hypothalamus, thalamus, and cortical areas, including the primary sensory cortex for the facial fields (Fig. 2).

While the final outcome of these changes has not been clearly established, there is experimental evidence for the role of the vagus and trigeminal nerves in regulating several physiologic pathways including: cerebral blood flow; melanocortins and inflammation; glutamate excitotoxicity; 


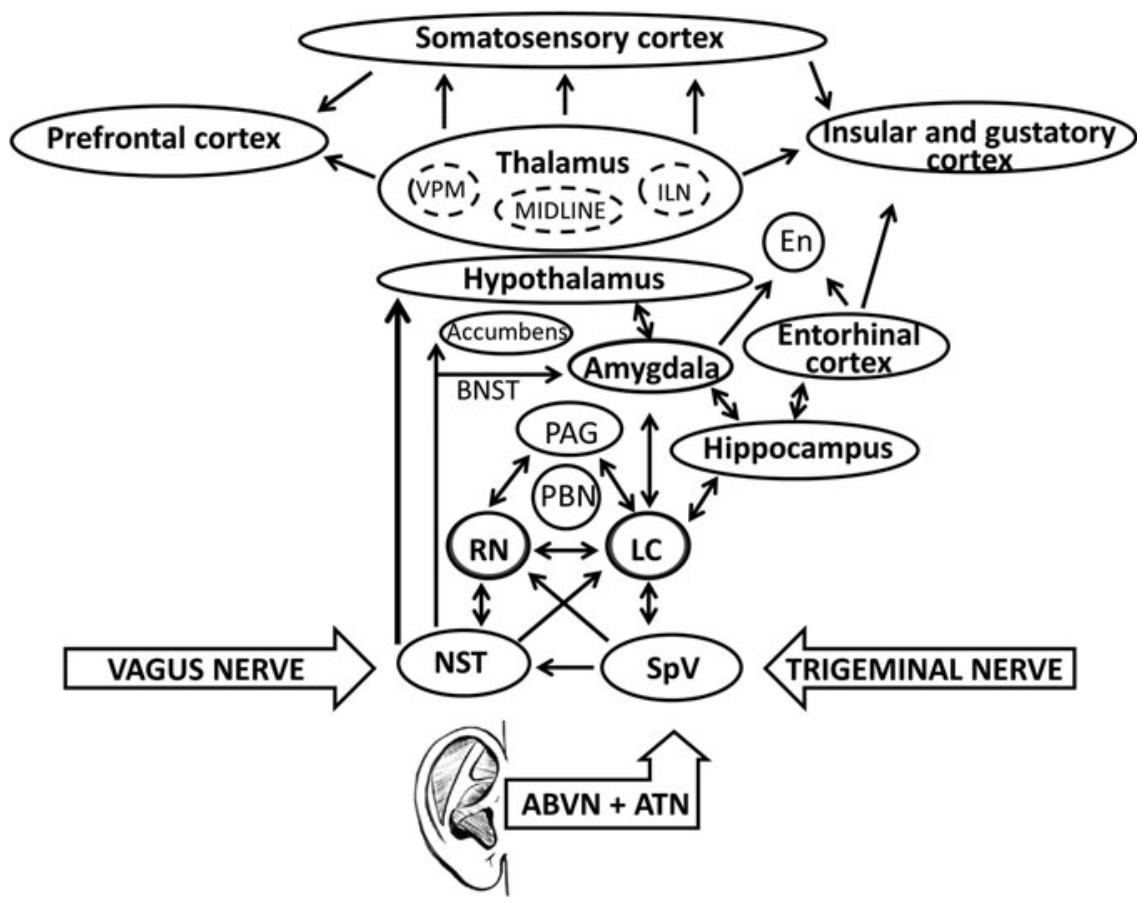

FIG. 2. Schematic representation of the main areas in the central nervous system areas possibly influenced by external ear stimulation via vagal and trigeminal nerves. VPM, ventral posterior medial nucleus; ILN, intralaminar nuclei; En, endopiriform nucleus; BNST, bed nucleus stria terminalis; PAG, periacquductal gray matter; PBN, nucleus parabrachialis; RN, raphe nucleus; LC, locus coeruleus; NST, nucleus of the solitarii tract; ABVN, auricular branch of the vagus nerve; ATN, auricular temporal branch of the trigeminal nerve.

neurotropic processes; and GABAegic, glutamatergic, and acetylcholinergic systems. All of these structures and processes are involved in the regulation of vigilance, mood, anxiety, cognitive processes, pain, food intake, metabolism, propensity to seizures, the sleep-wake cycle, hormone regulation, and reward/addiction.

A crucial point to be emphasized is that the mechanisms by which stimulation of the vagus and trigeminal nerves can exert central therapeutic effects is essentially an open question. Interestingly, scientific and clinical applications have largely preceded knowledge of the mechanism of action of peripheral nerve stimulation, which still presents a challenge for researchers. This is due, in part, to the lack of knowledge about the effects of such stimulation throughout the CNS and, in part, to the lack of knowledge of the pathologic mechanisms that underlie the treated disorders.

Indeed, some experimental researchers investigated the effects of electrical stimulation on animal models through several methods. However, all animal studies, so far, have been performed with little or no relationship to devices used with humans, and have not examined the effects of different stimulation parameters in detail, to help understand which treatment might produce a reliable and reproducible effect. Furthermore, there is still a lack of a functional map of the brain, which would help determine which sites of the brain to stimulate in order to elicit a predictable response.

Therefore, it is possible to affirm that, so far, experimental data have not produced practical effects with respect to the development of established neuromodulation protocols in humans. Yet, adequate animal models are necessary to understand how these treatments alleviate symptoms in humans and to explain the biologic effects of the stimulation devices and protocols currently used in humans.

\section{CONCLUSIONS}

A growing body of literature reports that auricular stimulation exerts therapeutic effects on several disorders and can restore the homeostatic balance in patients with dysfunctional conditions. Experimental evidence is increasingly supporting a role for the vagal and trigeminal afferent systems in the clinical effects described following auricular stimulation. These data have demonstrated that multiple areas in the CNS, which are of key importance to the pathophysiology of neuropsychiatric illnesses, could be targeted by vagal and trigeminal neuromodulation via stimulation of the external ear.

However, despite the wide knowledge of vagus and trigeminal nerves anatomy and physiology, the mechanisms responsible for the efficacy of VNS and TNS treatments are still poorly understood. Although some research examining their effects on animal models does exist, auricular neuromodulation deserves further mechanistic studies on its potentially favorable effects on physiologic biomarkers associated with neurophysiologic, 
neurotrophic, cerebrovascular, cardiovascular, and metabolic functions.

With this perspective, future research should also address the question regarding the possible relationships among the different protocols used currently in humans and the effects observed on the abovementioned biomarkers, to establish which stimulation parameters produce reliable and reproducible effects and to identify a core set of outcomes that best portray functional and clinical changes.

\section{AUTHOR DISCLOSURE STATEMENT}

No competing financial interests exist.

\section{REFERENCES}

1. Sale MV, Mattingley JB, Zalesky A, Cocchi L. Imaging human brain networks to improve the clinical efficacy of non-invasive brain stimulation. Neurosci Biobehav Rev. 2015;57:187-198.

2. Taghva AS, Malone DA, Rezai AR. Deep brain stimulation for treatment-resistant depression. World Neurosurg. 2012; 80(3-4):S27.e17-S27.e24.

3. Deng ZD, McClintock SM, Oey NE, Luber B, Lisanby SH. Neuromodulation for mood and memory: From the engineering bench to the patient bedside. Curr Opin Neurobiol. 2015;30:38-43.

4. Hoy KE, Fitzgerald PB. Brain stimulation in psychiatry and its effects on cognition. Nat Rev Neurol. 2010;6(5):267-275.

5. Albert GC, Cook CM, Prato FS, Thomas AW. Deep brain stimulation, vagal nerve stimulation and transcranial stimulation: An overview of stimulation parameters and neurotransmitter release. Neurosci Biobehav Rev. 2009;33(7):1042-1060.

6. Baeken C, Brunelin J, Duprat R, Vanderhasselt MA. The application of tDCS in psychiatric disorders: A brain imaging view. Socioaffect Neurosci Psychol. 2016;6(1):29588.

7. George MS, Aston-Jones G. Noninvasive techniques for probing neurocircuitry and treating illness: Vagus nerve stimulation (VNS), transcranial magnetic stimulation (TMS) and transcranial direct current stimulation (tDCS). Neuropsychopharmacology. 2009;35(1):301-316.

8. Ebajemito JK, Furlan L, Nissen C, Sterr A. Application of transcranial direct current stimulation in neurorehabilitation: The modulatory effect of sleep. Front Neurol. 2016;7:54.

9. Woods AJ, Antal A, Bikson M, et al. A technical guide to tDCS, and related non-invasive brain stimulation tools. Clin Neurophysiol. 2015;127(2):1031-1048.

10. Marder E, Thirumalai V. Cellular, synaptic and network effects of neuromodulation. Neural Netw. 2002;15(4-6):479493.

11. Lopez HS, Brown AM. Neuromodulation. Curr Opin Neurobiol. 1992;2(3):317-322.

12. Shiozawa P, da Silva ME, de Carvalho TC, Cordeiro Q, Brunoni AR, Fregni F. Transcutaneous vagus and trigeminal nerve stimulation for neuropsychiatric disorders: A systematic review. Arq Neuropsiquiatr. 2014;72(7):542-547.
13. Goroszeniuk T, Pang D. Peripheral neuromodulation: A review. Curr Pain Headache Rep. 2014;18(5):412.

14. Bailey P, Bremer F. A sensory cortical representation of the vagus nerve with a note on the effects of low blood pressure on the cortical electrogram. J Neurophysiol. 1938:1;405412.

15. Dell P, Olson R. Secondary mesencephalic, diencephalic and amygdalian projections of vagal visceral afferences [in undetermined language]. C R Seances Soc Biol Fil. 1951; 145(13-14):1088-1091.

16. MacLean PD. The Triune Brain in Evolution: Role in Paleocerebral Functions. New York: Plenum Press; 1990.

17. Zabara J. Peripheral control of hypersynchronous discharge in epilepsy. Electroencephalogr Clin Neurophysiol. 1985; 61:S162.

18. Zabara J. Time course of seizure control to brief, repetitive stimuli. Epilepsia. 1985;26(suppl5):518.

19. Zabara J. Inhibition of experimental seizures in canines by repetitive vagal stimulation. Epilepsia. 1992;33(6):10051012.

20. Ruffoli R, Giorgi FS, Pizzanelli C, Murri L, Paparelli A, Fornai $\mathrm{F}$. The chemical neuroanatomy of vagus nerve stimulation. J Chem Neuroanat. 2010;42(4):288-296.

21. Schachter SC. Vagus nerve stimulation therapy summary: Five years after FDA approval. Neurology. 2002;59(6[suppl4]): S15-S20.

22. Howland RH. Vagus nerve stimulation. Curr Behav Neurosci Rep. 2014;1(2):64-73.

23. Schachter SC, Saper CB. Vagus nerve stimulation. Epilepsia. 1998;39(7):677-686.

24. Sato T, Yamada N, Morimoto K, Uemura S, Kuroda S. A behavioral and immunohistochemical study on the development of perirhinal cortical kindling: A comparison with other types of limbic kindling. Brain Res. 1998;811(1-2): 122-132.

25. Goodnick PJ, Rush AJ, George MS, Marangell LB, Sackeim HA. Vagus nerve stimulation in depression. Expert Opin Pharmacother. 2001;2(7):1061-1063.

26. Gaynes BN, Farley JF, Dusetzina SB, et al. Does the presence of accompanying symptom clusters differentiate the comparative effectiveness of second-line medication strategies for treating depression? Depress Anxiety. 2011;28(11): 989-998.

27. Elger G, Hoppe C, Falkai P, Rush AJ, Elger CE. Vagus nerve stimulation is associated with mood improvements in epilepsy patients. Epilepsy Res. 2000;42(2-3):203-210.

28. Rush AJ, Marangell LB, Sackeim HA, Vagus nerve stimulation for treatment-resistant depression: A randomized, controlled acute phase trial. Biol Psychiatry. 2005;58(5): 347-354.

29. Nune G, DeGiorgio C, Heck C. Neuromodulation in the treatment of epilepsy. Curr Treat Options Neurol. 2015; 17(10):375.

30. Ben-Menachem E, Revesz D, Simon BJ, Silberstein S. Surgically implanted and non-invasive vagus nerve stimulation: A review of efficacy, safety and tolerability. Eur J Neurol. 2015;22(9):1260-1268.

31. Schuurman PR, Beukers RJ. Ventricular asystole during vagal nerve stimulation. Epilepsia. 2009;50(4):967-968. 
32. DeGiorgio CM, Murray $\mathrm{D}$, Markovic $\mathrm{D}$, Whitehurst $\mathrm{T}$. Trigeminal nerve stimulation for epilepsy: Long-term feasibility and efficacy. Neurology. 2009;72(10):936-938.

33. Fanselow EE, Reid AP, Nicolelis MA. Reduction of pentylenetetrazole-induced seizure activity in awake rats by seizure-triggered trigeminal nerve stimulation. $J$ Neurosci. 2000;20(21):8160-8168.

34. Loguidice C. Device Targeting Symptoms of Drug-Resistant Lennox-Gastaut Syndrome Receives Humanitarian Use Device Designation. January 22, 2015. Online document at: www .raredr.com/news/device-lgs-receives-humanitarian-use-devicedesignation Accessed August 11, 2017.

35. Schoenen J, Vandersmissen B, Jeangette S, Herroelen L, Vandenheede M, Gérard P, Magis D. Migraine prevention with a supraorbital transcutaneous stimulator: A randomized controlled trial. Neurology. 2013;80(8):697-704.

36. Trevizol AP, Shiozawa P, Sato IA, et al. Trigeminal nerve stimulation (TNS) for generalized anxiety disorder: A case study. Brain Stimul. 2014;8(3):659-660.

37. Cao B, Wang J, Shahed M, Jelfs B, Chan RH, Li Y. Vagus nerve stimulation alters phase synchrony of the anterior cingulate cortex and facilitates decision making in rats. $S c i$ Rep. 2016;6:35135.

38. Wang Q-Q, Zhu L-J, Wang X-H, et al. Chronic trigeminal nerve stimulation protects against seizures, cognitive impairments, hippocampal apoptosis, and inflammatory responses in epileptic rats. J Mol Neurosci. 2016;59(1): 78-89.

39. Sjögren MJ, Hellström PT, Jonsson MA, Runnerstam M, Silander HC, Ben-Menachem E. Cognition-enhancing effect of vagus nerve stimulation in patients with Alzheimer's disease: A pilot study. J Clin Psychiatry. 2002;63(11):972980.

40. Jin Y, Kong J. Transcutaneous vagus nerve stimulation: A promising method for treatment of autism spectrum disorders. Front Neurosci. 2017;10:609.

41. Soleymani T, Pieton D, Pezeshkian P, Miller P, Gorgulho AA, Pouratian N, De Salles AA. Surgical approaches to tinnitus treatment: A review and novel approaches. Surg Neurol Int. 2011;2(1):154.

42. Engineer ND, Riley JR, Seale JD, et al. Reversing pathological neural activity using targeted plasticity. Nature. 2011;470(7332):101-104.

43. Hays SA, Ruiz A, Bethea T, et al. Vagus nerve stimulation during rehabilitative training enhances recovery of forelimb function after ischemic stroke in aged rats. Neurobiol Aging. 2016;43:111-118.

44. Chiluwal A, Narayan RK, Chaung W, et al. Neuroprotective effects of trigeminal nerve stimulation in severe traumatic brain injury. Sci Rep. 2017;7(1):6792.

45. Dawson J, Pierce D, Dixit A, et al. Safety, feasibility, and efficacy of vagus nerve stimulation paired with upper-limb rehabilitation after ischemic stroke. Stroke. 2015;47(1):143150.

46. Trevizol AP, Shiozawa P, Sato IA, et al. Trigeminal nerve stimulation (TNS) for post-traumatic stress disorder: A case study. Brain Stimul. 2015;8(3):676-678.

47. McClelland J, Bozhilova N, Campbell I, Schmidt U. A systematic review of the effects of neuromodulation on eating and body weight: Evidence from human and animal studies. Eur Eat Disord Rev. 2013;21(6):436-455.

48. Göbel CH, Tronnier VM, Münte TF. Brain stimulation in obesity. Int J Obes (Lond). 2017;41(12):1721-1727.

49. Mei N, Condamin M, Boyer A. The composition of the vagus nerve of the cat. Cell Tissue Res. 1980;209(3):423431.

50. Foley JO, DuBois FS. Quantitative studies of the vagus nerve in the cat: I. The ratio of sensory to motor fibers. $J$ Comp Neurol. 1937;67(1):49.

51. Agostoni E, Chinnock JE, Daly MBD, Murray JG. Functional and histological studies of the vagus nerve and its branches to the heart, lungs and abdominal viscera in the cat. J Physiol (Lond). 1957;135(1):182-205.

52. Magerl W, Ali Z, Ellrich J, Meyer RA, Treede RD. C- and A-delta-fiber components of heat-evoked cerebral potentials in healthy human subjects. Pain. 1999;82(2):127-137.

53. Iannetti GD, Leandri M, Truini A, Zambreanu L, Cruccu G, Tracey I. Adelta nociceptor response to laser stimuli: Selective effect of stimulus duration on skin temperature, brain potentials and pain perception. Clin Neurophysiol. 2004; 115(11):2629-2637.

54. Mercante B, Enrico P, Floris G, et al. Trigeminal nerve stimulation induces Fos immunoreactivity in selected brain regions, increases hippocampal cell proliferation and reduces seizure severity in rats. Neuroscience. 2017;361:69-80.

55. Mercante B, Pilurzi G, Ginatempo F, et al. Trigeminal nerve stimulation modulates brainstem more than cortical excitability in healthy humans. Exp Brain Res. 2015;233(11): 3301-3311.

56. Pilurzi G, Mercante B, Ginatempo F, Follesa P, Tolu E, Deriu F. Transcutaneous trigeminal nerve stimulation induces a long-term depression-like plasticity of the human blink reflex. Exp Brain Res. 2015;234(2):453-461.

57. Ginatempo F, Pirina P, Melis F, Deriu F. Short-term trigeminal neuromodulation does not alter sleep latency in healthy subjects: A pilot study. Neurol Sci. 2017;September 30:e-pub ahead of print.

58. Cook IA, Espinoza R, Leuchter AF. Neuromodulation for depression: Invasive and noninvasive (deep brain stimulation, transcranial magnetic stimulation, trigeminal nerve stimulation). Neurosurg Clin N Am. 2013;25(1):103-116.

59. DeGiorgio CM, Krahl SE. Neurostimulation for drug-resistant epilepsy. Continuum (Minneap Minn). 2013;19(3[Epilepsy]): 743-755.

60. Fanselow EE. Central mechanisms of cranial nerve stimulation for epilepsy. Surg Neurol Int. 2012;3(supp14):S247-S254.

61. Pop J, Murray D, Markovic D, DeGiorgio CM. Acute and long-term safety of external trigeminal nerve stimulation for drug-resistant epilepsy. Epilepsy Behav. 2011;22(3):574-576.

62. Soss J, Heck C, Murray D, et al. A prospective long-term study of external trigeminal nerve stimulation for drugresistant epilepsy. Epilepsy Behav. 2014;42:44-47.

63. DeGiorgio CM, Shewmon A, Murray D, Whitehurst T. Pilot study of trigeminal nerve stimulation (TNS) for epilepsy: A proof-of-concept trial. Epilepsia. 2006;47(7):1213-1215.

64. Topchiy I, Radulovacki M, Waxman J, Carley DW. Cardiorespiratory effects of intertrigeminal area stimulation in vagotomized rats. Brain Res. 2009;1250:120-129. 
65. Samuels ER, Szabadi E. Functional neuroanatomy of the noradrenergic locus coeruleus: Its roles in the regulation of arousal and autonomic function. Part II: Physiological and pharmacological manipulations and pathological alterations of locus coeruleus activity in humans. Curr Neuropharmacol. 2009;6(3):254-285.

66. Aston-Jones G, Shipley MT, Chouvet G, et al. Afferent regulation of locus coeruleus neurons: Anatomy, physiology and pharmacology. Prog Brain Res. 1991;88:47-75.

67. Fornai F, Ruffoli R, Giorgi FS, Paparelli A. The role of locus coeruleus in the antiepileptic activity induced by vagus nerve stimulation. Eur J Neurosci. 2011;33(12):2169-2178.

68. Chae J-H, Nahas Z, Lomarev M, et al. A review of functional neuroimaging studies of vagus nerve stimulation (VNS). J Psychiatr Res. 2003;37(6):443-455.

69. Henry TR. Therapeutic mechanisms of vagus nerve stimulation. Neurology. 2002;59(6[supp14]):S3-S14.

70. Giorgi FS, Pizzanelli C, Biagioni F, Murri L, Fornai F. The role of norepinephrine in epilepsy: from the bench to the bedside. Neurosci Biobehav Rev. 2004;28(5):507-524.

71. Grimonprez A, Raedt R, Baeken C, Boon P, Vonck K. The antidepressant mechanism of action of vagus nerve stimulation: Evidence from preclinical studies. Neurosci Biobehav Rev. 2015;56:26-34.

72. Marrosu F, Serra A, Maleci A, Puligheddu M, Biggio G, Piga M. Correlation between GABA(A) receptor density and vagus nerve stimulation in individuals with drug-resistant partial epilepsy. Epilepsy Res. 2003;55(1-2):59-70.

73. Walker BR, Easton A, Gale K. Regulation of limbic motor seizures by GABA and glutamate transmission in nucleus tractus solitarius. Epilepsia. 1999;40(8):1051-1057.

74. Vertes RP, Fortin WJ, Crane AM. Projections of the median raphe nucleus in the rat. J Comp Neurol. 1999;407(4):555-582.

75. Manta S, Dong J, Debonnel G, Blier P. Enhancement of the function of rat serotonin and norepinephrine neurons by sustained vagus nerve stimulation. J Psychiatry Neurosci. 2009;34(4):272-280.

76. Dorr AE, Debonnel G. Effect of vagus nerve stimulation on serotonergic and noradrenergic transmission. $J$ Pharmacol Exp Ther. 2006;318(2):890-898.

77. Follesa P, Biggio F, Gorini G, et al. Vagus nerve stimulation increases norepinephrine concentration and the gene expression of BDNF and bFGF in the rat brain. Brain Res. 2007;1179:28-34.

78. Biggio F, Gorini G, Utzeri $\mathrm{C}$, et al. Chronic vagus nerve stimulation induces neuronal plasticity in the rat hippocampus. Int Neuropsychopharmacol. 2009;12(9):1209-1221.

79. Revesz D, Tjernstrom M, Ben-Menachem E, Thorlin T. Effects of vagus nerve stimulation on rat hippocampal progenitor proliferation. Exp Neurol. 2008;214(2):259-265.

80. Santarelli L, Saxe M, Gross C, et al. Requirement of hippocampal neurogenesis for the behavioral effects of antidepressants. Science. 2003;301(5634):805-809.

81. Zuo Y, Smith DC, Jensen RA. Vagus nerve stimulation potentiates hippocampal LTP in freely-moving rats. Physiol Behav. 2007;90(4):583-589.

82. Grimonprez A, Raedt R, Portelli J, et al. The antidepressantlike effect of vagus nerve stimulation is mediated through the locus coeruleus. J Psychiatr Res. 2015;68:1-7.
83. Vonck K, Raedt R, Naulaerts J, et al. Vagus nerve stimulation ... 25 years later! What do we know about the effects on cognition? Neurosci Biobehav Rev. 2014;45:63-71.

84. Cai PY, Bodhit A, Derequito R, et al. Vagus nerve stimulation in ischemic stroke: Old wine in a new bottle. Front Neurol. 2014;5:107.

85. Bonaz B, Sinniger V, Pellissier S. Anti-inflammatory properties of the vagus nerve: Potential therapeutic implications of vagus nerve stimulation. J Physiol (Lond). 2016;594(20): 5781-5790.

86. Bodenlos JS, Schneider KL, Oleski J, Gordon K, Rothschild AJ, Pagoto SL. Vagus nerve stimulation and food intake: Effect of body mass index. J Diabetes Sci Technol. 2014; 8(3):590-595.

87. Banni S, Carta G, Murru E, et al. Vagus nerve stimulation reduces body weight and fat mass in rats. PLoS One. 2012; 7(9):e44813.

88. DeGiorgio CM, Fanselow EE, Schrader LM, Cook IA. Trigeminal nerve stimulation: Seminal animal and human studies for epilepsy and depression. Neurosurg Clin N Am. 2011;22(4):449-456.

89. He W, Wang X, Shi H, Shang H, Li L, Jing X. Auricular acupuncture and vagal regulation. Evid Based Complement Alternat Med. 2012;2012:786839.

90. Peuker ET, Filler TJ. The nerve supply of the human auricle. Clin Anat. 2002;15(1):35-37.

91. Kreuzer PM, Landgrebe M, Resch M, et al. Feasibility, safety and efficacy of transcutaneous vagus nerve stimulation in chronic tinnitus: An open pilot study. Brain Stimul. 2014;7(5):740-747.

92. Kraus T, Hösl K, Kiess O, Schanze A, Kornhuber J, Forster C. BOLD fMRI deactivation of limbic and temporal brain structures and mood enhancing effect by transcutaneous vagus nerve stimulation. J Neural Transm (Vienna). 2007; 114(11):1485-1493.

93. Zhao YX, He W, Jing XH, et al. Transcutaneous auricular vagus nerve stimulation protects endotoxemic rat from lipopolysaccharide-induced inflammation. Evid Based Complement Alternat Med. 2012;2012:627023.

94. Kraus T, Kiess O, Hösl K, Terekhin P, Kornhuber J, Forster C. CNS BOLD fMRI effects of sham-controlled transcutaneous electrical nerve stimulation in the left outer auditory canal—a pilot study. Brain Stimul. 2013;6(5):798-804.

95. Hein E, Nowak M, Kiess O, et al. Auricular transcutaneous electrical nerve stimulation in depressed patients: A randomized controlled pilot study. J Neural Transm (Vienna). 2012;120(5):821-827.

96. Laqua R, Leutzow B, Wendt M, Usichenko T. Transcutaneous vagal nerve stimulation may elicit anti- and pronociceptive effects under experimentally-induced pain-a crossover placebo-controlled investigation. Auton Neurosci. 2014;185:120-122.

97. Rong P-J, Fang J-L, Wang L-P, et al. Transcutaneous vagus nerve stimulation for the treatment of depression: A study protocol for a double blinded randomized clinical trial. BMC Complement Altern Med. 2012;12:255.

98. Rong P, Liu A, Zhang J, et al. An alternative therapy for drug-resistant epilepsy: Transcutaneous auricular vagus nerve stimulation. Chin Med J. 2014;127(2):300-304. 
99. Polak T, Markulin F, Ehlis A-C, Langer JBM, Ringel TM, Fallgatter AJ. Far field potentials from brain stem after transcutaneous vagus nerve stimulation: Optimization of stimulation and recording parameters. J Neural Transm (Vienna). 2009;116(10):1237-1242.

100. Weise D, Adamidis M, Pizzolato F, Rumpf J-J, Fricke C, Classen J. Assessment of brainstem function with auricular branch of vagus nerve stimulation in Parkinson's disease. PLoS One. 2015;10(4):e0120786.

101. Fallgatter AJ, Neuhauser B, Herrmann MJ, et al. Far field potentials from the brain stem after transcutaneous vagus nerve stimulation. J Neural Transm (Vienna). 2003;110(12): 1437-1443.

102. Frangos E, Ellrich J, Komisaruk BR. Non-invasive access to the vagus nerve central projections via electrical stimulation of the external ear: fMRI evidence in humans. Brain Stimul. 2014;8(3):624-636.

103. Fang J, Rong P, Hong $\mathrm{Y}$, et al. Transcutaneous vagus nerve stimulation modulates default mode network in major depressive disorder. Biol Psychiatry. 2015;79(4):266-273.

104. Ma J, Zhang L, He G, Tan X, Jin X, Li C. Transcutaneous auricular vagus nerve stimulation regulates expression of growth differentiation factor 11 and activin-like kinase 5 in cerebral ischemia/reperfusion rats. J Neurol Sci. 2016;369: 27-35.

105. Hays SA, Khodaparast N, Hulsey DR, Ruiz A, Sloan AM, Rennaker RL 2nd, Kilgard MP. Vagus nerve stimulation during rehabilitative training improves functional recovery after intracerebral hemorrhage. Stroke. 2014;45(10):3097-3100.

106. Chen M, Yu L, Ouyang F, Liu Q, et al. The right side or left side of noninvasive transcutaneous vagus nerve stimulation: Based on conventional wisdom or scientific evidence? Int $J$ Cardiol. 2015;187:44-45.

107. da Silva MAH, Dorsher PT. Neuroanatomic and clinical correspondences: Acupuncture and vagus nerve stimulation. J Altern Complement Med. 2013;20(4):233-240.

Address correspondence to

Franca Deriu, MD, PhD

Department of Biomedical Sciences

University of Sassari

Viale San Pietro 43/b

07100-Sassari Italy

E-mail: deriuf@uniss.it 